\title{
Implementation of Intelligent Wireless Sensor Network using Geo-Casting Routing
}

\author{
Rekha Kak \\ Baddi University of Emerging Science and \\ Technologies (BUEST) \\ Baddi, Himachal Pradesh, India
}

\author{
Abhishek Gupta \\ Baddi University of Emerging Science and \\ Technologies (BUEST) \\ Baddi, Himachal Pradesh, India
}

\begin{abstract}
Wireless sensor network (WSN) is the basic and interesting areas among practitioners and researchers in various fields. As sensor nodes are prone to environmental changes and external agents. That's why it is becoming necessary to organize the wireless sensor network which is secure, energy efficient and has prolonged network life. The organization of sensors in wireless sensor network system plays a considerable role in network performance such as sensing, communication and connectivity. The main objective is to consider incompatible objectives in order to expand the network coverage and minimize the energy consumption of the network. This manuscript focuses on a specialized algorithmic approach for the deployment of wireless sensor nodes so that the network is energy efficient. In this manuscript, a unique algorithm is proposed as well as implemented that spotlight on energy optimization factor. The energy competence of the sensor nodes are managed by dipping the bandwidth usage by avoiding probing of other sensor nodes for communication causes. The energy loss in sensor node in our approach was greatly reduced. By comparing the results derived after simulation it is uncovered that our methodology out performs in terms of energy consumption, packet loss, throughput and jitter.
\end{abstract}

Keywords - Wireless Sensor Nodes, Energy Optimization, Deployment Scheme

\section{INTRODUCTION}

The study and deep exploration of wireless sensor networks is demanding huge span of knowledge from huge variety of disciplines. The improvement and usage of wireless sensor networks was aggravated by military applications like battleground surveillance, industrial process monitoring, consumer applications and health monitoring control mechanism. The wireless sensor networks draws attention to several restrictions like: Sensor nodes are closely placed in limited range areas where competent multi-hop routing, energy efficient and secured algorithms are required. Sensor networks are unpredictable, prone to collisions and the structure of network changes repeatedly. Therefore it is desirable to setup the energy controlled multi-path routing. These devices are having limited power capacity, low processing power and limited memory which emphasises the topology or design control to such that minimum transmission power is required. The node deployment in the sensor network depends entirely on the kind of demand and directly effects the output of the methods implemented. It generally needs to discover the ideal bunching that permits the best network. Since the communication between nodes is generally constrained in transfer speed and the packet delivery time, the most possible courses might be structured by multi- hop wireless ways. Sensor nodes may be in any of these states i.e. sensing, relaying, sleeping and dead.

Energy utilization in the sensor hubs are arranged into the three parts, i.e. Energy for the sensor transducer, Energy for correspondence among sensor hubs and Energy for the microchip calculation. From the study it is found that every bit which is being transmitted in the WSNs devours to the extent of processing in 800-1000 directions. Accordingly, the correspondence is more immoderate than the processing in WSN. Any message extension brought about by security components takes a stab at a critical expense of power consumption. Further, higher security levels in WSN normally relate to more energy utilization for cryptographic capacities. Consequently, the WSN could be separated into distinctive security levels contingent upon the energy cost .The embedded computing devices in sensor nodes are generally not as powerful as those in nodes of a wired network. As such, typical cryptographic algorithms cannot be used in WSN's.

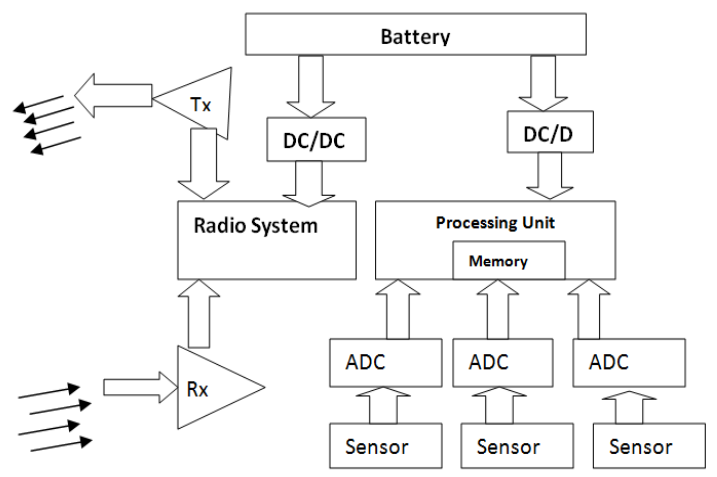

Fig 1:View of a Sensor Node

A node is made up of a power supplying system, comprising of a battery and DC-DC converters as per requirement, a processing system consisting of a processor and memory, the physical parameter sensing sensors and transceiver system comprising of a transmitter and a receiver.

However, due to the vigorous changes of actions and unreceptive environment a pure static WSN could face the severe problems. Sensor nodes dropped from the aircraft or by robots could not guarantee efficient connectivity to cover the area completely to be sensed. To overcome this problem, area need to partition into various non-connected sub networks. Moreover, the vibrant change of area of interest and the presence of obstacles could add to the problems. Sensor nodes are usually battery-powered and prone to errors. Holes in WSN get created due to the death of certain nodes because of their battery consumption. These death nodes may break the network connectivity. It is quite difficult to recharge sensor nodes or deploy new nodes to replace these death nodes. The 
WSN may be required to support multiple missions under various conditions. For example, in an object tracking application, sufficient sensor nodes should be deployed along the track of the target, while in a boundary detection mission; there should be adequate nodes along the pre-described perimeter. These different requirements cannot be easily satisfied by deploying a large amount of sensor nodes, since provisioning for all possible combinations of mission requirements could not be economically feasible. Some applications may need sophisticated sensors to involve in. For example, one can image that in a military surveillance application, pressure sensors are used along the boundary to detect whether any enemy intrudes in. However, these sensors can only detect and report about something passing through but cannot identify what passes through them. In this case, more sophisticated sensing devices like cameras should be required to obtain more information. Nevertheless, it is infeasible to equip camera on each node because of their large number. Advantages of suitable sensor propagation in WSNs are : energy efficiency, low cost, routing backbone i.e. by deployment of cluster heads, scalability, collision reduction, load sharing, increased connectivity and less delay, minimum cluster heads, maximum network life.

\subsection{Characteristics and Features}

The main characteristics of a WSN which require optimization at different layers to fulfill application requirements include:

Power utilization constraints for nodes using batteries, event management, fault tolerance and ease of use need be optimization at operating system level.

Data sensing, collection and reporting methods while ensuring Quality of Service, query broadcasting, heterogeneity of nodes and network dynamics require to be optimized at network layer.

Load sharing, throughput and power/energy optimization at Data link layer.

At component level parameter like processor voltage, computation capability, sensing frequency and modulation schemes need to be tuned.

Mobility of nodes and scalability to expand node deployment area, connectivity, coverage and ability to cope with node failures, all these parameters require optimization at architectural level.

\section{PROPOSED APPROACH AND METHODOLOGY}

WSNs are intended to be deployed in various environments comprising of far-flung and unreceptive regions where ad hoc communications plays a major role. Energy consumption, conservation and optimization are major challenges in wireless sensor networks particularly in outlining a directing convention. Therefore, the energy efficient protocols and paradigms are the major area of active research to focus on the issues like lifetime maximization, robustness, fault tolerance and self-configuration. The critical as well as crucial requirement in wireless sensor node is that it should be capable of collecting and transferring necessary details to centralized monitoring system. As the wireless sensor nodes are low battery powered nodes, the power or energy backup is affected when its performance gets improved and vice versa. In order to overcome this problem an intelligent sensor network has been introduced in this manuscript. The novel intelligent sensor network follows the advance methodologies to overcome the problem such as communication failure, battery consumption, shortest route to transfer packet to destination, monitoring by centralized system and ability to find new path in case of any link failure and trusted communication .Energy is the rare resource as the lifetime of WSN is entirely dependent on it. By introducing mobility to some or all nodes in a WSN, we can enhance its capability and flexibility to support multiple missions and to handle the fore mentioned problems. Although a WSN is usually considered as an ad hoc network in which nodes are extended with sensing capability. Mobility in a mobile ad hoc network is often subjective to random changes, as compared to mobility in a mobile WSN should be "premeditated". In other words, we can control the movement of mobile sensors to conduct different missions. Wireless Sensor Network can be self-organized into network and transmit nodes information. To conserve power the node should shut off the radio power supply when not in use. The proposed work moves the sensors independently and collects the information in various scenarios, and these sensors also are able to replace the failed sensor nodes and gap between the nodes to collect the information in energy efficient manner. The results were compared with the previously implemented techniques. In the existing techniques data reduction strategy is one of the schemes employed to extend network lifetime. Existing techniques presented an implementation of a light-weight forecasting algorithm for sensed data which saves packet transmission in the network. This approach achieves the high energy optimization with an overhead of limited computational on a node with following disadvantages:

There is no shortest route to transfer packet to destination.

Existing technique is not energy efficient.

Existing technique will not be able to find new path in case of any link failure.

Existing technique is open to the intruders (attackers).

The main objective of the proposed work is, wireless sensor node should be capable of collecting and transferring necessary details to centralized monitoring system. In WSNs, a base station is a wireless communication station installed at a fixed location to communicate and used to gather data from other nodes. The sink base station is used to communicate with the user via satellite communication. It is located very near to the sensor field or well equipped nodes of the sensor network. In this proposed methodology the sensor nodes are arranged into groups, each group should be provided with head sensor node. The head sensor node passes the relevant details of other nodes to the base station. The base station in turn passes this information to server or central monitoring system. In case the sensor node tries to transfer packet to other sensor node to perform some action, in our approach it follows the optimized technique. Nowadays the sensor nodes are equipped with advanced features like GPS, so the tracking of sensor node is much easier than the earlier techniques. The location specific details are transferred to server through header node and base station. The energy efficiency of the sensor nodes are managed by reducing the bandwidth usage, so by avoiding the searching of another sensor nodes for communication causes the energy loss in sensor node, in our approach is greatly reduced. 


\section{Advantage}

It follows shortest route to transfer packet to destination.

It is energy efficient.

It finds new path in case of any link failure.

It is trusted and free from intruders (attackers).

Its details are be monitored by centralized system

\section{Disadvantage}

Follows advanced platform for implementation.

Location information may be incorrect in case of obstruction like tunnel or under hills under real practical scenarios.

\section{RESULTS OF EXISTING / PROPOSED APPROACH}

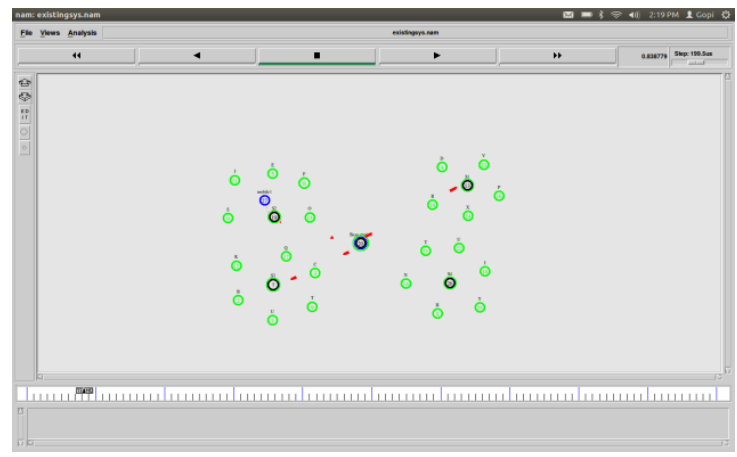

Fig 2 : Displays position of 27 nodes, connection established between normal nodes and mobile nodes with base station in the classical approach.

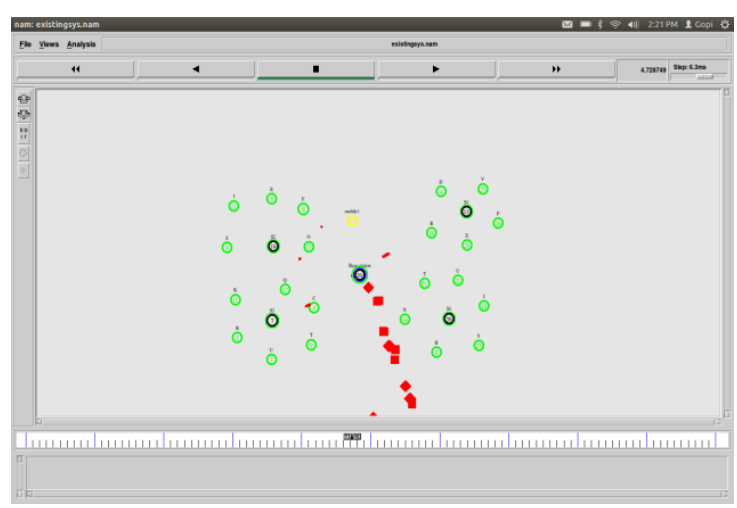

Fig 3 : When mobile node moves away from the network, packet drop will occur due to lack of connectivity. If packets are forwarded to receiver at that time receiver is not in range to accept the packets and will cause the drop of packets.

\subsection{Implementation of the Proposed}

In the proposed work the mobile receiver node is prevented from moving away from the network as it was monitored by satellite and that information is passed to the base station via header node. So the base station gives command to the sensor node to change the travelling direction from current path.

\subsection{Proposed Algorithmic Flow}

1.Initialize Wireless Nodes (Wi) [ Wi <= n ] [n:Max.

No. Deployable Nodes]

2. Deployment and Configuration of Sensor Nodes

3. Initialize BSi [Activation of Base Station]

4. Activation of MN[i] -> Mobile Nodes

5.MN $[\mathrm{i}]<=>B S[i]$ [Conn. Established] CEts (Time Stamp Recorded)

6. Set d [Distance Vector] -> Threshold

7. if $\operatorname{dist}(\mathrm{MN}[\mathrm{i}], \mathrm{BS}[\mathrm{i}])>=\mathrm{d}$ (Threshold)

$\operatorname{di}[\mathrm{m}]=>$ packet drop matrix under process

GoTo Step 1

8. Else

if NOT(dist(MN[i], BS[i])

$\mathrm{ACK}[\mathrm{i}]=>\mathrm{BS}[\mathrm{ACK}] \Rightarrow$ Activation and transmission of Acknowledgement Vector

9.SN[SatelliteNode ] $\Leftrightarrow$ Vi $\Leftrightarrow$ MN[i] (Satellite Monitors Mobile Node Movement)

10. DPI [DataPacketInfo] $=>\mathrm{SA}=>\mathrm{BS}[\mathrm{i}]$

11. Measurement dist(MN[i], BS[i])

12.R[j][Restriction Vector] [Avoidance of PacketDrop[k]]

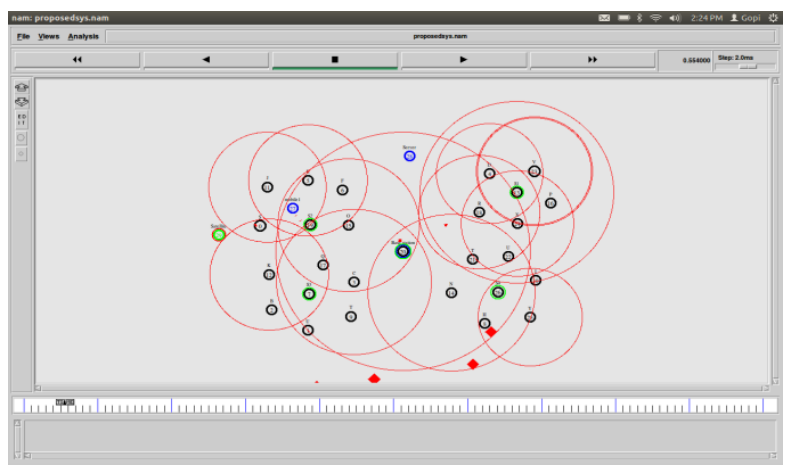

Fig 4 : Displays Position of 29 nodes in proposed approach while nodes are sending acknowledgments to base station and Satellite node monitoring Mobile 1's node position

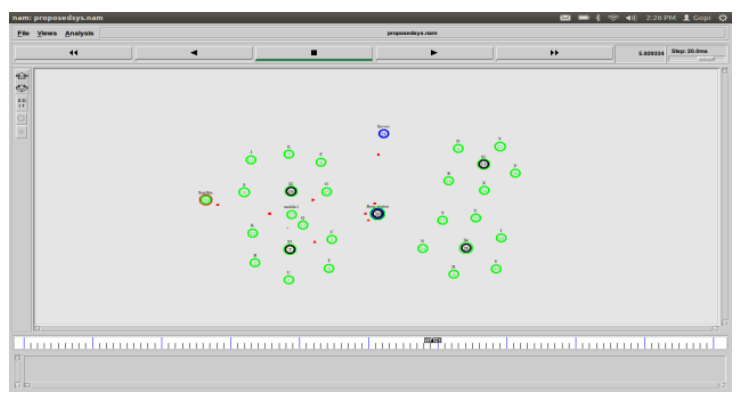

Fig 5 : Mobile node 1 passes information to server through base station. Mobile node 1 is prevented from moving away from network and forced to stay connected with the network which results in reduction of packet drop.

\subsection{Comparative Graph and Performance Evaluation}

Existing packet loss $\quad$ Proposed packet loss

615




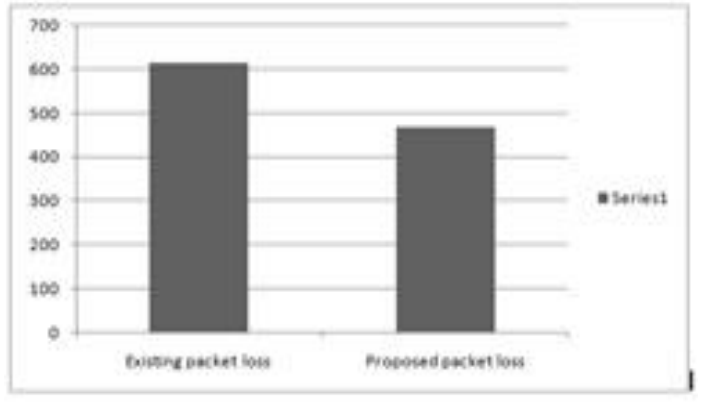

Fig 6 : Packet loss comparison

The existing technique simulation result shows the packet drop higher than proposed

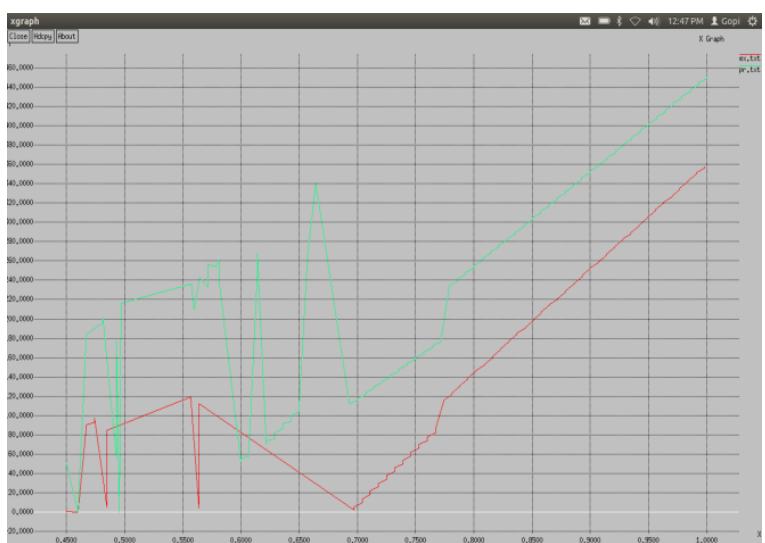

Fig 7 - Packet Transfer Rate

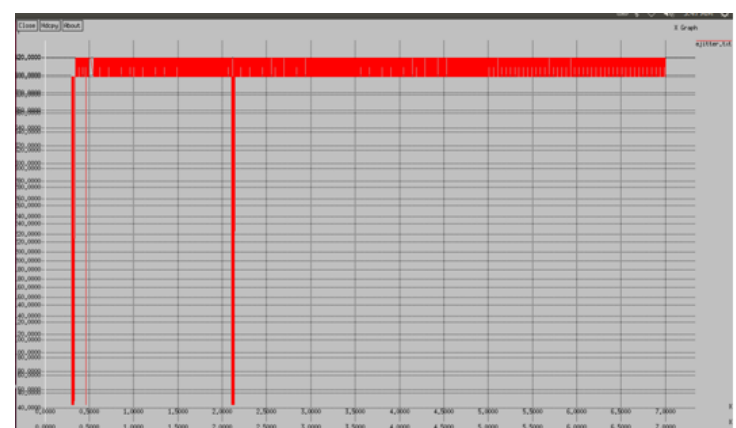

Fig 8 : Jitter Parameter Analysis (Existing Approach)

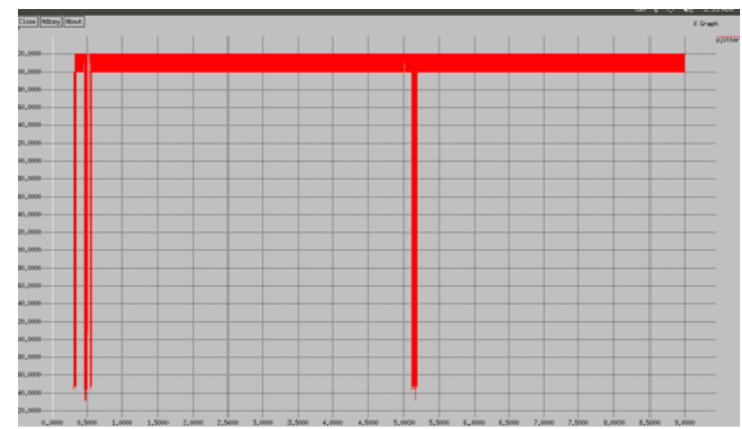

Fig9 : Jitter Parameter Analysis (Proposed Approach)

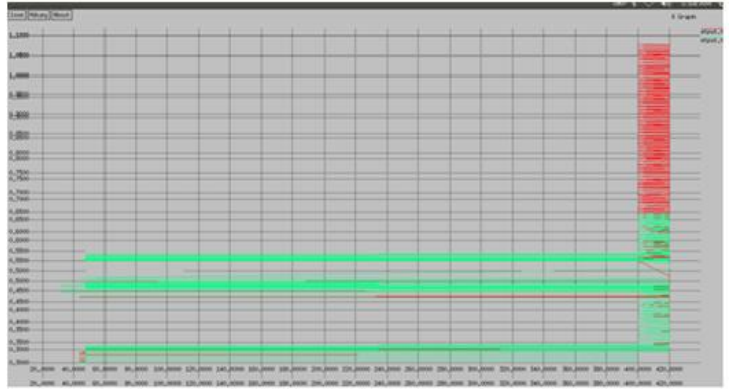

Fig 10 : Throghput Parameter Analysis (Existing and Proposed Approach)

\section{CONCLUSION AND FUTURE WORK}

In this thesis a deployment approach is proposed where, the wireless sensor nodes are able to move independently, the relay nodes are used connect the nodes to provide maximum efficiency. The proposed work move the sensors independently and collect the information in various scenarios, and these sensors also are able to replace the failed sensor nodes and gap between the nodes to collect the information in energy efficient manner. The results were compared with the previously implemented techniques. In the thesis work the mobile receiver node is prevented from moving away from the network as it was monitored by satellite and that information is passed to the base station via header node. So the base station gives command to the sensor node to change the travelling direction from current path. Proposed methodology is better in throughput, jitter and is energy efficient than classical approach.

The simulation and deployment techniques could be proposed appropriate for both static and mobile Ad hoc WSNs. Also, more user interface services could be added to give the users supplementary flexibilities for selecting the proper limitations which suits their own examined algorithms.

\section{REFERENCES}

[1] . N. Trigoni, Y. Yao, A. Demers, J. Gehrke, and R. Rajaramany, "Wave scheduling: Energy-efficient data dissemination for sensor networks," in Proceedings of the International Workshop on Data Management for Sensor Networks (DMSN), International Confernece on Very Large Data Bases (VLDB), August 2004.

[2 ] Sandra Sendra, Jaime Lloret, Miguel García and José F. Toledo "Power saving and energy optimization techniques for Wireless Sensor Networks". Journal of Communication, Vol. 6, No. 6, September 2011.

[3] Antoine B. Bagula and Kuzamunu G. Mazandu F.E. Sandnes, "Energy Constrained Multipath Routing in Wireless Sensor Networks," pp. 453-467, 2008, Springer-Verlag Berlin Heidelberg.

[4] Power saving and energy optimization techniques for Wireless Sensor Networks, Journal Of Communications, VOL. 6, NO. 6, September 2011

[5] S. Saxena, S. Mishra, A. Kumar and D. S. Chauhan, "Efficient Power Utilization Techniques for Wireless Sensor Networks-A Survey", International Journal on Computer Science and Engineering, vol.:3, Issue:2, Pp. 905-925, $7^{\text {th }}$ Annual IEEE communication Society conference, February 2011. 
[6] "An Energy Distribution and Optimization Algorithm in Wireless Sensor Networks for Maritime Search and Rescue," International Journal of Distributed Sensor Networks, Volume 2013, Article ID 725869.

[7] Mohanad Al-Obaidi, "De Montfort Energy Optimization Algorithm for Mobile Wireless Sensor Networks using Evolutionary Computation and Swarm Intelligence", Ph. D thesis, De-Montfort University, 2010

[8] Zeynep Orman1, Ali Norouzi, Faezeh Sadat Babamir, "Intelligent Node Placement using GA (INPGA)
Protocol in Wireless Sensor Networks," Proceedings of the International Conference on Wireless Networks (ICWN'11), 2011.

[9] Anjali, Navpreet Kaur,"Optimization of Energy in Wireless Sensor Networks", International Journal of Engineering Trends and Technology, Volume4 ,Issue32013

[10] "Energy Optimization Approaches In Wireless Sensor Networks", International Journal of Networks and Systems, Volume 1, No.1, July August- September 2012 